\title{
Genomic variation among closely related Vibrio alginolyticus strains is located on mobile genetic elements
}

\section{Cynthia Maria Chibani}

Christian-Albrechts-Universitat zu Kiel

Heiko Liesegang

Georg-August-Universitat Gottingen

\section{Olivia Roth}

Helmholtz-Zentrum fur Ozeanforschung Kiel

Carolin Charlotte Wendling ( $\nabla$ carolin.wendling@env.ethz.ch )

Eidgenossische Technische Hochschule Zurich https://orcid.org/0000-0003-4532-7528

\section{Research article}

Keywords: pangenome, mega-plasmids, mobile genetic elements, horizontal gene transfer, genomic islands, niche-adaptation

Posted Date: February 17th, 2020

DOI: https://doi.org/10.21203/rs.2.23653/v1

License: (9) This work is licensed under a Creative Commons Attribution 4.0 International License. Read Full License

Version of Record: A version of this preprint was published at BMC Genomics on May 11th, 2020. See the published version at https://doi.org/10.1186/s12864-020-6735-5. 


\section{Abstract}

Background Species of the genus Vibrio, one of the most diverse bacteria genera, have undergone niche adaptation followed by clonal expansion. Niche adaptation and ultimately the formation of ecotypes and speciation in this genus has been suggested to be mainly driven by horizontal gene transfer (HGT) through mobile genetic elements (MGEs). Our knowledge about the diversity and distribution of Vibrio MGEs is heavily biased towards human pathogens and our understanding of the distribution of core genomic signatures and accessory genes encoded on MGEs within specific Vibrio clades is still incomplete. We used nine different strains of the marine bacterium Vibrio alginolyticus isolated from pipefish in the Kiel-Fjord to perform a multiscale-comparative genomic approach that allowed us to investigate (1) those genomic signatures that characterize a habitat-specific ecotype and (2) the source of genomic variation within this ecotype.

Results We found that the nine isolates from the Kiel-Fjord have a closed-pangenome and did not differ based on core-genomic signatures. Unique genomic regions and a unique repertoire of MGEs within the Kiel-Fjord isolates suggest that the acquisition of gene-blocks by HGT played an important role in the evolution of this ecotype. Additionally, we found that $\sim 90 \%$ of the genomic variation among the nine isolates is encoded on MGEs, which supports ongoing theory that accessory genes are predominately located on MGEs and shared by HGT. Lastly, we could show that these nine isolates share a unique virulence and resistance profile which clearly separates them from all other investigated $V$. alginolyticus strains and suggests that these are habitat-specific genes, required for a successful colonization of the pipefish, the niche of this ecotype.

Conclusion We conclude that all nine $V$. alginolyticus strains from the Kiel-Fjord belong to a unique ecotype, which we named the Kiel-alginolyticus ecotype. The low sequence variation of the core-genome in combination with the presence of MGE encoded relevant traits, as well as the presence of a suitable niche (here the pipefish), suggest, that this ecotype might have evolved from a clonal expansion following HGT driven niche-adaptation.

\section{Background}

The genomic variation within a bacterial species is reflected by accessory genes. Together with strainspecific singletons (genes found in only one strain) and the core-genes that are shared by all strains of a species (such as housekeeping genes and genes required for essential cellular functions) the accessory elements form the pangenome (1). Accessory genes are usually only found in a subset of genomes of a species and are attributed to habitat-specific functions, such as strain-specific virulence factors (2), antibiotic resistance (3), or niche-adaptation (4). As such, accessory genes are often located on mobile genetic elements (MGEs), including plasmids, bacteriophages, transposons, integrative or conjugative elements (ICEs) and genomic islands (GIs) and are shared between species via horizontal gene transfer (HGT) (5). Even though HGT between strains of the same species is more frequent, some MGEs have a 
broad host range enabling them to move genes across species boundaries thereby blurring the definition of a species (6).

Not only the size of pangenomes differs among species but also the relationship between the proportion of core-genes relative to the pangenome (7). A large pangenome with a proportionally small core-genome and with high rates of HGT is considered open, whereas a small pangenome with a high proportion of core-genes and low HGT rates is considered to be closed (8). For a wide range of bacteria, the pangenome size is positively correlated with the effective population size $\left(\mathrm{N}_{\mathrm{e}}\right)(7)$. Species with a high $\mathrm{N}_{e}$ are likely to occupy different environmental niches where they experience a larger diversity of selection pressures, which will drive selection for increased genome diversity and ultimately lead to larger pangenomes (8). However, niche adaptation in species with high $\mathrm{N}_{e}$ can also select for distinct ecotypes (i.e. ecologically and evolutionary distinct subpopulations) and ultimately initiate a speciation process with a niche-specific pangenome (8). It remains debated when ecotypes are sufficiently diverged to be considered different species (9).

An excellent model organism to study pan-core genomic signatures and niche adaptation resulting from HGT is the genus Vibrio. These bacteria constitute one of the most diverse bacterial genera with a large pangenome comprising more than 100 species from different clades, that occupy different ecological niches $(10,11)$, where they can exist as pathogenic, opportunistic, mutualistic or free-living forms (12). The considerable large pangenome ( $>26,500$ genes) of the genus Vibrio, which is more than 50 times larger than the Vibrio specific core-genome (11), indicates a large reservoir of genomic diversity for this genus. In contrast, within a specific Vibrio clade, the pangenome to core-genome ratio decreases considerably. For instance, within the cholerae clade, the pangenome is only 4.5 times larger than the core-genome (11). The relatively small cholerae-pangenome (6923 genes) compared to the entire Vibriopangenome, might reflect a specific set of genes, required to occupy the cholera-specific niche.

Niche adaptation and ultimately speciation in this genus has been suggested to be mainly driven by HGT (13). Furthermore, MGEs, in particular filamentous phages, contribute significantly to the emergence of pathogenic vibrios from environmental populations (6) and the prophage repertoire of a given Vibrio species can account for a large fraction of the variation among strains $(14,15)$. Our knowledge about the diversity and distribution of Vibrio MGEs is heavily biased towards human pathogens, e.g. V. cholerae and V. parahaemolyticus. While recent studies started to expand our knowledge on Vibrio-specific MGEs to other clades, such as V. anguillarum $(16,17)$, V. fischerii (18), or V. alginolyticus (19), our understanding about the distribution of core-genomic signatures and accessory genes encoded on MGEs within specific Vibrio clades is still incomplete. This might be because most of the available genomes are draft genomes comprising multiple contigs, whereas only a few complete genomes of environmental vibrios are available to date (19). It is challenging to generate high-quality genomes of vibrios due to repetitive genomic regions such as rRNA operons present in the two chromosomes (8-12) and arrays of multiple integrated prophages. Especially multiple prophage arrays cannot be resolved using draft genome assemblies from short-read sequencing techniques. Due to the incomplete information concerning the 
genome organization stored in draft genomes, it is not possible to study genome dynamics of mobile genetic elements especially of prophages without long-read sequencing data (20).

The present study aimed to investigate how genome organization, as well as core-genomic signatures and accessory genes, differ within a group of closely related environmental Vibrio isolates. To do so, we performed a multi-scale comparative genomics approach using Vibrio alginolyticus as a model organism. V. alginolyticus, a ubiquitous marine opportunistic pathogen can cause mass mortalities in shellfish, shrimp, and fish, resulting in severe economic losses worldwide $(3,21,22)$. Vibrio pathogenicity is a complex interaction of abiotic and biotic factors, including high temperatures $(23,24)$ low salinities $(25)$, host and bacterial genotypes $(26,27)$. To date only six closed alginolyticus genomes are available, while 28 remain fragmented with 2 to 75 contigs (last accessed: December 2019). A detailed comparative genomic characterization of the alginolyticus species, including plasmid content, distribution of MGEs and virulence factors of this species is as yet missing. Here we analysed core-and accessory-genomic signatures of $\mathrm{V}$. alginolyticus by comparing nine closely related $\mathrm{V}$. alginolyticus genomes, isolated from the pipefish Syngnathus typhle in the Kiel-Fjord (28), (later named Kiel-alginolyticus ecotype) with less closely related $\mathrm{V}$. alginolyticus isolates (Table 1).

\section{Results And Discussion}

\section{Genome features of Vibrio alginolyticus isolated from the Kiel-Fjord}

We sequenced the genomes of nine Vibrio alginolyticus strains, previously isolated from pipefish in the Kiel-Fjord (28) using a combination of PacBio long- and Illumina short-read technology. The assembly resulted in eight closed genome sequences and one draft genome (strain K09K1), where both chromosomes have been assembled into a single contig due to multiple copies of an integrated filamentous phage. The replicon boundaries could not be resolved experimentally based on PCR thus strain $\mathrm{K} 09 \mathrm{~K} 1$ has been assigned a "permanent draft" status. All V. alginolyticus genomes contain a 3.47 Mbp chromosome 1 and a $1.88 \mathrm{Mbp}$ chromosome 2, with a \%GC content of around 44\% (Table 1), which is typical for the genus Vibrio as well as for the species V. alginolyticus $(45,46)$. We found extrachromosomal replicons including plasmids and filamentous phages in seven isolates (Table 1).

\section{Species definition and phylogenetic relationship of the Kiel- Fjord isolates}

All nine strains isolated from the Kiel-Fjord share more than $98 \%$ average nucleotide identity (ANI) with other alginolyticus strains, suggesting that these strains belong, as previously suggested based on a multi-locus-sequencing-approach (MLSA) (23), to the species V. alginolyticus. All alginolyticus strains share $~ 92 \%$ ANI with two other closely related Vibrio species, i.e. V. diabolicus and V. antiquarius (Fig. 1), which is below the species threshold of $A N I=95 \%(47,48)$, indicating that $\mathrm{V}$. alginolyticus is a distinct species. 


\section{The Vibrio alginolyticus pan/ core genome}

Pangenome: To compare the gene repertoire of the nine V. alginolyticus strains from the Kiel-Fjord with the global V. alginolyticus gene repertoire, we calculated their pangenome including the amount of coreand accessory genes as well as strain-specific singletons, i.e. genes unique to single strains (49). The analysis included in total 73,277 protein-sequences encoded on chromosomes and MGEs, including plasmids and extra-chromosomal phage replicons. Overall, we found that the size of the pangenome of V. alginolyticus increased stronger (from 4997 to 8843 gene clusters) with the sequential addition of each new genome when we included all 15 strains from diverse habitats (Fig. 2a). In contrast, we only observed a slight increase in the pangenome (from 5044 to 5679 gene clusters) when we only included the nine V. alginolyticus isolates from the Kiel-Fjord (Fig. 2b). An increase in the pangenome results from new strain-specific genes, found in each newly analyzed isolate. The much larger increase in the pangenome across all available $\mathrm{V}$. alginolyticus isolates relative to the increase in the pangenome of KielFjord isolates suggests that the genomic diversity of V. alginolyticus within the Kiel-Fjord is very limited. However, the species V. alginolyticus per se has a vast reservoir of genomic diversity.

We extrapolated both, the within-Kiel and the global V. alginolyticus pangenome by fitting a least-squares curve based on Heaps' Law (1) and found that the within-Kiel pangenome is closed $(a=1.12)$, whereas the global V. alginolyticus pangenome is open $(a=0.58)$. This means, that within the global V. alginolyticus pangenome each newly sequenced $\mathrm{V}$. alginolyticus isolate will reveal a unique set of genes, irrespective of the number of strains included in the present analysis. This open $\mathrm{V}$. alginolyticus pangenome reflects the diversity in habitats in which this species exists. These distinct environments probably require different adaptations and/ or promote high levels of HGT. In contrast, a closed pangenome, as has been detected for the within-Kiel V. alginolyticus pangenome, suggests that the number of genes that will be obtained from any newly sequenced isolate will converge to zero. Here, we assume, that the at least sequenced pipefish associated V. alginolyticus strains within the Kiel-Fjord contain the major part of the gene equipment that is requested to adapt to their habitat. Thus the genomes can be expected to be highly similar, potentially resulting from niche adaptation and strong selection thereof. Strong positive selection of such adaptive genes might have led to a clonal expansion of the Kiel-alginolyticus ecotype. Indeed, we found no sequence divergence based on core-genomic signature among all nine isolates from Kiel (Fig. 2C), indicating a clonal expansion of this ecotype. It remains to be investigated, whether free-living $\mathrm{V}$. alginolyticus strains or isolates from other eukaryotic hosts from the Kiel-Fjord share the same gene-pool or are more divergent from the pipefish-associated strains.

Core-genome and singletons: We observed a stronger decrease in the core-genome when we included all $V$. alginolyticus isolates as opposed to when we performed the analysis only within the Kiel-alginolyticus ecotype. Comparative analyses between all $15 \mathrm{~V}$. alginolyticus strains and the Kiel-alginolyticus ecotype only, revealed that the core genome (4708 gene clusters) is four times larger than the accessory genome (971 gene clusters) when we only included the nine Kiel strains. However, when we extended the analysis and included all $15 \mathrm{~V}$. alginolyticus, we found that the core genome (3876 gene clusters) becomes smaller 
than the accessory gene pool (4967 gene clusters). In other words, within the Kiel-Fjord, different V. alginolyticus isolates have a large core-genome ( $83 \%$ of the pangenome) with a limited accessory gene pool (17\% of the pangenome). In contrast, despite all being members of the same species, the global accessory gene-pool is highly variable ( $56 \%$ of the pangenome) and the V. alginolyticus core-genome constitutes only $44 \%$ of the pangenome.

\section{Habitat specific chromosomal regions}

The acquisition of entire gene-blocks (genomic islands, plasmids, prophages) by HGT can rapidly alter the life-style of a bacterium in quantum leaps (50). This mechanism seems to be particularly important for bacterial adaptation to new ecological niches but also for how bacteria diverge from each other, forming ecotypes and ultimately new species (51). Genomic islands, which encode specific functions allowing for niche adaptation and maybe even speciation events are common within the genus Vibrio (52). For the Kiel-alginolyticus ecotype, we could identify 19 chromosomal genomic regions (GRs) of which most are unique to the strains isolated from the Kiel-Fjord (Fig. 3, Table S2). Overall, these 19 GRs encode a total of 487 genes out of which 305 have only been found within the Kiel-alginolyticus ecotype. Out of these 19 GRs five could be assigned to integrated prophages. GR 13 and GR 14 correspond to Vibrio phage VALG 2 2/2b and Vibrio phage VALGФ6, which are unique for the isolates from the Kiel-Fjord and have not been found elsewhere (19). GR 3 corresponds to Vibrio phage VALG $\Phi 1$, which has so far only been detected in V. alginolyticus ATCC33787, with a relatively low query cover of $57 \%$, thus making it a unique region for Kiel. In contrast GR 5, which corresponds to the filamentous phage Vibrio phage VALG $\Phi 8$ on chromosome 1, is absent in most Kiel strains, except K10K4 and K05K4, but present in some non-Kiel strains, such as ATCC17749 and FDAARGOS_114, suggesting that GR 5 is not unique for the Kiel isolates. Similarly, GR 15, which corresponds to a multiphage-cassette consisting of multiple repeats of a combination of the two filamentous phages Vibrio phage VALG $\$ 6$ and Vibrio phage VALGФ8 (19), is absent in most Kiel strains, except K04M3 and K04M5 but in parts present in ATCC17749 and thus not unique for the Kiel system. Of the remaining $14 \mathrm{GRs}$, which do not correspond to integrated phages, we could identify four genomic islands: GR 2, GR 6, GR 7 and GR 8 (> $10 \mathrm{~kb}$, presence of integrase/ transposase, different GC content). According to functional COG categories, these islands contain a variety of proteins, most of them involved in replication, recombination, and repair [L] and transcription $[\mathrm{K}]$ (see Table S3 for functional annotation of all GRs). The high prevalence of those maintenance genes is however not surprising and might result from an identification bias, as they are usually better annotated for MGEs than accessory genes, which would provide a selective advantage to the host. As such, many proteins were classified as phage integrases, transposases or other proteins, involved with viral integration into host DNA or DNA repair, suggesting that HGT might have played an important role during the acquisition of these Gls. GR 6 encodes the multi-drug transporter subunit MdtN and two other loci which were assigned to COG category [V] and predicted to be involved in defense mechanisms. This suggests that GR 6 might play a role in resistance. None of the other genomic islands could be identified as pathogenicity-/ metabolic- or resistance island. All other GRs were either smaller than $10 \mathrm{~kb}$ or did not contain integrases or transposases and are thus referred to as genomic regions instead of genomic island. Out of these GRs, GR 16 encoded three proteins associated with the type VI secretion system and 
GR 18 encoded a beta-lactamase protein suggesting an adaptive role in virulence and resistance, respectively. The acquisition of these unique GRs might have allowed the Kiel-alginolyticus ecotype to invade a new niche, which was then followed by clonal expansion of this ecotype. Clonal expansion has been observed for several pathogens, in particular within the genus Vibrio. The best characterized example of clonal expansion upon acquisition of virulence genes is V. cholerae. But also other Vibrio pathogens, for instance $V$. parahaemolyticus have been shown to experience similar evolutionary dynamics (for a review see (53)).

\section{Genomic differences within Kiel-alginolyticus ecotype}

To investigate genomic differences between the nine strains from the Kiel-Fjord, we focused on all those gene-clusters from the Roary analysis, which could only be found within the Kiel-alginolyticus ecotype but were absent in all non-Kiel isolates. We found that all Kiel-specific core-genes $(n=412)$ were located exclusively on one of the two chromosomes (Fig. 4). In contrast, the majority of the Kiel-specific accessory gene clusters $(89 \%)$ were encoded on mobile genetic elements, in particular plasmids. These results support ongoing theory that accessory genes are predominately located on MGEs and shared by horizontal gene transfer (HGT) (5). We found 490 gene clusters with no orthologous in any other Kiel strain, i.e. singletons out of which $40 \%$ were located on MGEs, in particular, extrachromosomal replicating elements (170 on plasmids and 27 on extrachromosomal phages) and $60 \%(n=293)$ were chromosomal (Fig. 4). All Kiel-specific alginolyticus gene clusters were further assigned to putative functional categories using the Clusters of Orthologous Groups of Proteins (COG) database (34) (Table S4). Even though a large fraction of the gene clusters could either not be assigned to a COG or was poorly characterized, we found differences in the relative distribution of super-functional COGs between coreand accessory genomes and singletons: The majority of the singletons (37\%) was predicted to be dedicated to cellular processes/ signaling, while relatively small proportions of gene clusters $(10 \%$ and $16 \%$ ) belong to information storage/ processing and metabolism. In contrast, the largest proportion of gene-clusters encoded on core-genes was predicted to belong to information storage/ processing (24\%), and only $16 \%$ and $13 \%$ of gene-clusters encoded on core genes belong to cellular processes/ signaling and metabolism. Among the gene-clusters encoded on the accessory genome $22 \%$ could be assigned to information storage/ processing as well as to cellular processes/ signaling and only $6 \%$ to the metabolism (Fig. 4). Within the accessory genome most of the genes are involved in replication, recombination, and repair (COG [L], Table S4). These include mainly proteins involved in HGT, such as transposases, integrases, transferases, recombinases as well as proteins involved in immunity, such as CRISPR associated helicase Cas3 and restriction modification methylases. This extensive representation of proteins involved in HGT on the accessory genome suggests that HGT was potentially one of the driving evolutionary mechanisms underlying the diversification of the nine $\mathrm{V}$. alginolyticus strains from the Kiel-Fjord.

The majority of the accessory gene-pool within the Kiel-alginolyticus ecotype is located on plasmids (Fig. 4). We could identify four different plasmids from all nine Kiel V. alginolyticus isolates. Three plasmid types isolated from the Kiel strains were unique for specific strains with a size of 0.9 to $2.9 \mathrm{kbp}$ 
(Table 1). The fourth plasmid type was characterized as a mega-plasmid (Fig. 5a), which ranged between 280 and $300 \mathrm{kbp}$ in size, and was present in six out of the nine strains (Fig. 5b). The six mega-plasmids share 296 core-genes, encode 129 accessory genes and between 5-26 singletons per plasmid (Fig. 5b). Together with the three small plasmids, plasmid-encoded singletons make up $34.5 \%$ of all 486 Kielspecific singletons. The majority of the plasmid-specific singletons comprise hypothetical proteins. The remaining singletons include AAA proteins (K04M1_pL280), PFAM phosphoadenosine phosphosulfate reductase and ATPases (K04M5_pL294), spore germination and type IV secretion system (K5K4_pL289), endonuclease and site-specific methyl-transferase, potentially forming a restriction-modification system (K06K5_pL291), and DNA polymerase (K08M3_pL300). V. alginolyticus ATCC 33787 contains as well three plasmids including plasmid pMBL287, which is similar in size as the Kiel mega plasmids (46). However, a comparison of ATCC 33787 plasmids revealed no sequence similarity to any of the plasmids from the Kiel strains.

Only $6.5 \%$ of the 870 Kiel-specific accessory genes and singletons are located on extrachromosomal phages. The within-Kiel variation caused by these phages can be explained by the absence of extrachromosomal replicons of Vibrio phage VALG $\$ 8$ in all but two strains (K04M1 and K05K4). However, four strains (K04M3, K04M5, K05K4, and K10K4) had an intra-chromosomal version of this phage, while strains K01M1, K06K5 and K08M3 did neither contain an intra- nor an extrachromosomal version of Vibrio phage VALGФ8 (for a detailed analysis of Kiel vibriophages see (19)). Parts of Vibrio phage VALGФ8 could be identified on both chromosomes of ATCC $17749^{\top}$, and on chromosome 1 of FDAARGOS_114 but not on any other non-Kiel Vibrio. Genome analyses of Vibrio phage VALG $\$ 8$ revealed no significant virulence-associated genes nor any other genes that could be associated with a habitatspecific adaptation (19).

\section{Virulence and resistance of the Kiel-Fjord V. alginolyticus isolates}

We found an identical virulence and resistance profile among the nine $\mathrm{V}$. alginolyticus isolates from the Kiel-Fjord. The Kiel-Fjord isolates encode a total number of 17 homologues resistance genes out of which the majority $(n=13)$ is located on chromosome 1 and four resistance genes are encoded on chromosome 2 (Fig. 6, Table S5). In comparison with other V. alginolyticus strains ( 25-130 resistance genes on Chromosome 1 and 11-12 resistance genes on Chromosome 2), isolates from the Kiel-Fjord have significantly less resistance genes (t-test: $\mathrm{t}_{8.85}=3.51, \mathrm{p}=0.007$; Fig. 6 ) and are missing in particular genes conferring resistance to tetracycline, aminoglycosides, and quinolones.

In contrast to non-pathogenic strains, such as V. fischerii, which is a mutualist containing 112 virulence genes, the Kiel-Fjord isolates encode more virulence genes (150 virulence genes, exception strain K05K4: 149 virulence genes). This number is lower than what has been found for human pathogenic vibrios, such as V. cholerae ( 165 virulence genes) and V. parahaemolyticus (162 virulence genes), but similar to what has been found in other V. alginolyticus strains, except (strain FDAARGOS_114: 165 virulence genes). Unique for the Kiel-Fjord isolates is the absence of genes involved in Vibriobactin biosynthesis, 
which are present in almost all other V. alginolyticus strains. Similarly, In contrast to other V. alginolyticus isolates, the strains from the Kiel-Fjord miss the gene flaC, which is involved in the regulation of stringentresponse-induced toxin production (54) and vscP, a gene involved in the type III secretion system. In contrast to other V. alginolyticus strains, the Kiel-alginolyticus ecotype encodes the type IV secretion system effectors, the phytotoxin coronatine and the thermolabile hemolysin (tlh), both toxins, which could not be found in any other $\mathrm{V}$. alginolyticus strain. This unique virulence profile of the Kiel-Fjord isolates separates them from other Vibrio species including other $\mathrm{V}$. alginolyticus isolates abut also another strain from the Kiel-Fjord: V. typhli, K08M4 (55) (Fig. 6). This clear separation was further supported by a hierarchical cluster analysis (Fig. 6), which indicates, that only based on the presence/absence of virulence genes the Kiel-alginolyticus ecotype can be distinguished from all other $\mathrm{V}$. alginolyticus strains, sequenced to date. The unique resistance and virulence profile within the Kiel-Fjord V. alginolyticus isolates might be a further indication for niche-adaptation followed by clonal expansion of this ecotype.

\section{Conclusions}

By using a multi-scale comparative genomic approach, we conclude that the nine $\mathrm{V}$. alginolyticus strains isolated from pipefish in the Kiel-Fjord belong to a unique ecotype, which evolved from a clonal expansion following niche adaptation. We came to this conclusion based on the following observations. First, the nine strains from the Kiel-Fjord have a closed-pangenome reflecting low genomic diversity within its niche. Second, even though these strains have been isolated from different eukaryotic host species, they did not differ based on core-genomic signatures which has been previously suggested based on MLSA (23). Third, niche adaptation and ultimately speciation in the genus Vibrio have been suggested to be mainly driven by HGT (13). Likewise, unique GRs and a unique repertoire of MGEs within the Kiel-Fjord isolates (including unique plasmids and prophages) support ongoing theory that the acquisition of geneblocks by HGT, one of the most important mechanisms driving the evolution of niche adaptation and clonal expansion also played an important role in the evolution of the Kiel-alginolyticus ecotype. Lastly, the unique virulence and resistance profile which clearly separates the Kiel-Fjord isolates from all other investigated V. alginolyticus strains, suggests, that these are essential genes, required for a successful colonization of the pipefish, the niche of the Kiel-alginolyticus ecotype. To ultimately verify this theory, colonization experiments with knock-out mutants lacking the Kiel-specific virulence genes, or alternatively non virulent Vibrio strains equipped with the Kiel-specific virulence genes, would be required.

We are aware that our conclusion is limited by the low number of non-Kiel V. alginolyticus strains available included in the comparative genomic analyses. We could have included draft genomes, to increase the number of sequences available for comparison. However, due to the incomplete information especially for MGEs stored in draft genomes, the number of core-genomes drops considerably by approximately $50 \%$ compared to when only complete genomes are included in the analysis (20). In favor of a more accurate analysis, we thus decided to only include complete genomes.

The present study extends our current knowledge of pan-and core-genomic signatures in the genus Vibrio which is currently heavily biased towards human pathogens, such as V. cholerae and V. 
parahaemolyticus. Based on the present results we suggest that also non-human pathogens might experience similar evolutionary dynamics, once relevant pathogenic traits (in the present case: Vibrio phage VALGФ6 (19), plus three unique virulence genes (Fig. 6)) have been acquired. The availability of a suitable niche, in the present case, presumably the pipefish, might have led to niche adaptation and clonal expansion of the Kiel-alginolyticus ecotype, clearly separating it from other $\mathrm{V}$. alginolyticus species.

\section{Methods}

\section{Bacterial genome data}

Using a combination of PacBio and Illumina sequencing, we generated eight closed genomes and one permanent draft genome of nine Vibrio strains isolated from different pipefish in the Kiel-Fjord (28) and previously characterized as V. alginolyticus based on multi-locus-sequence typing (23). We additionally sequenced prophages induced with mitomycin $C$ from each strain, for details see (19). We compared all replicons from the nine $V$. alginolyticus strains to 10 closed Vibrio genome sequences downloaded from the NCBI nucleotide database; date of accession 1.12.2019 (Table S1).

\section{Comparative genome analysis}

\section{Average nucleotide identity}

The average similarity between the nine $\mathrm{V}$. alginolyticus genomes from the present study, six other complete V. alginolyticus genomes as well as four other closely related vibrios (i.e. three V. diabolicus and one $\mathrm{V}$. antiquarius) was measured as the average nucleotide identity (ANI) of all 19 Vibrio replicons (concatenated Chromosome 1 and Chromosome 2) using the ANIm mode from the PyANI python module (29) based on MUMmer (https://github.com/widdowquinn/pyani). Briefly, nucleotide sequences were extracted from each GenBank file using Biopython (https://biopython.org/) and subsequently used as input for PyANI for genome sequence alignment.

\section{Pan-Core genome prediction}

To determine the total gene-pool of V. alginolyticus within the Kiel-Fjord as well as the global gene repertoire of $\mathrm{V}$. alginolyticus, we performed two pan-core genome analyses. In the first analysis, we only included the nine strains from the Kiel-Fjord. In the second analysis, we included all fully closed V. alginolyticus genomes available from NCBI (accession date 10.10. 2019) which were isolated from various regions (Table 1), in addition to the nine Kiel strains. Genbank files of all closed V. alginolyticus isolates were converted to GFF3 files and a nucleotide alignment was generated using Roary 3.8.0 (30) with standard settings (minimum BLASTP identity of $90 \%$ with paralogue splitting). We used concatenated files for each isolate including both chromosomes and if existing, mobile genetic elements (plasmids and episomal phages). To estimate whether we have an open or a closed pangenome we used Heaps' Law: $n-k * N^{a}(1)$, implemented in the R package 'micropan' (31), where $\mathrm{h}$ is the expected number 
of genes for a given number of Genomes $(\mathrm{N})$, and $\mathrm{k}$ and a are constants to fit the specific curve. The exponent $a$ is an indicator whether the pangenome is open $(a<1)$ or closed $(a>1)$. Lastly, a functional characterization of all protein-coding sequences was performed using the eggnog-mapper v2 database v5.0 $(32,33)$. We subsequently determined the COG category (Cluster of Orthologous Groups of Proteins (34)) for Kiel-specific core- and accessory genes as well as singletons identified by the pan-core analysis based on the Roary clustering input table.

\section{Phylogenetic analysis}

To further infer the phylogenetic relationship among all V. alginolyticus isolates we generated a phylogenetic tree based on the core-genome alignment for all 15 strains obtained from the Roary analysis (length for all strains $3.84 \mathrm{Mbp}$, which corresponds to approximately $72 \%$ of the average size of the input genomes). To do so, we used the Bayesian Markov chain Monte Carlo (MCMC) method as implemented in MrBayes version 3.2.5 $(35,36)$. The TN93 $(37)$ model plus invariant sites $($ TN93 +1$)$, as suggested by the Akaike information criterion (AIC) given by jModelTest (38), was used as a statistical model for nucleotide substitution. The MCMC process was repeated for $10^{6}$ generations and sampled every 5000 generations. The first 2000 trees were deleted as burn-in processes and the consensus tree was constructed from the remaining trees. Convergence was assured via the standard deviation of split frequencies $(<0.01)$ and the potential scale reduction factor (PSRF 1). The resulting phylogenetic tree and associated posterior probabilities were illustrated using FigTree version 1.4.2 (http://tree.bio.ed.ac.uk/software/figtree/).

\section{Genomic Islands}

We used IslandViewer (39) to predict putative genomic islands of all nine isolates from the Kiel-Fjord. The selection criterion for genomic islands was based on the presence of mobile-related genes (integrases or transposases), a size $>8 \mathrm{~kb}$, and a different GC content compared to the rest of the genome. To visualize the genomic islands, we used BLAST Ring Image Generator $(40,41)$ and created a circular map per chromosome of all fully closed V. alginolyticus genomes. Functional characterization of all proteincoding sequences found on the putative genomic islands was performed as described above using the eggNOG-mapper v2 database v5.0 $(32,33)$ where the resulting COG assignments were used for further analysis.

\section{Plasmids}

We used Easyfig (42) for pairwise plasmid sequence comparisons and synteny comparisons with an Evalue cut-off of $1 e-10$. Plasmid maps were generated using SnapGene Viewer (version 4.3.10) with annotated genbank files as input files. The output resulting from Roary was used to determine orthologous genes, while the output of eggNOG-mapper $(32,33)$ was used to assign a functional characterization to the coding protein sequences of the six mega-plasmids detected in the Kiel-specific V. alginolyticus isolates.

\section{Virulence and resistance}


The virulence of the nine Kiel-isolates was determined using controlled infection experiments in a previous study (19), and could in part be explained by the presence and the coverage of a virulence-gene carrying filamentous phage Vibrio phage VALGФ6. To determine other potential chromosomal encoded virulence factors, we scanned all $15 \mathrm{~V}$. alginolyticus strains and seven other strains from different Vibrio clades using the Virulence Factor Database (https://www.mgc.ac.cn/VFs/main.htm) (43). We used IslandViewer (39) to identify homologues of resistance genes in all $15 \mathrm{~V}$. alginolyticus strains.

All statistics and visualization graphs were performed using the ggplot2 library (44) in R 3.1.2 unless otherwise stated.

\section{Abbreviations}

genomic islands (GIs), genomic regions (GRs), average nucleotide identity (ANI), multi-locus-sequencingapproach (MLSA), Clusters of Orthologous Groups of Proteins (COG) mobile genetic elements (MGEs), horizontal gene transfer (HGT)

\section{Declarations}

Ethics approval and consent to participate: Not applicable

Consent for publication: Not applicable

Availability of data and materials: The genomic sequences of the nine Kiel $V$. alginolyticus strains analyzed during the current study are available at GenBank (CP017889-CP017919).

Competing interests: The authors declare that they have no competing interests

Funding: This project was funded by three consecutive DFG grants [WE 5822/ 1-1, WE5822/1-2, and RO R0462/4-2] within the priority programme SPP1819 given to given to CCW and OR, a grant from the Cluster of Excellence "The Future Ocean", given to CCW, and a KAAD stipend given to CCM.

Authors' contributions: CCW, HL and OR designed the study. CMC and CCW analyzed the data. CCW wrote the manuscript. All authors commented on the manuscript and approved the final version.

Acknowledgements: We are grateful to Anja Poehlein for her support in sequencing the isolates.

\section{References}

1. Tettelin H, Riley D, Cattuto C, Medini D. Comparative genomics: the bacterial pan-genome. Curr Opin Microbiol. 2008;11(5):472-7.

2. Rasko DA, Rosovitz MJ, Myers GSA, Mongodin EF, Fricke WF, Gajer P, et al. The pangenome structure of Escherichia coli: Comparative genomic analysis of E-coli commensal and pathogenic isolates. $J$ Bacteriol. 2008;190(20):6881-93. 
3. Aagesen AM, Phuvasate S, Su YC, Hase CC. Persistence of Vibrio parahaemolyticus in the Pacific Oyster, Crassostrea gigas, Is a Multifactorial Process Involving Pili and Flagella but Not Type III Secretion Systems or Phase Variation. Appl Environ Microb. 2013;79(10):3303-5.

4. Austin B, Austin DA, Blanch AR, Cerda M, Grimont F, Grimont PAD, et al. A comparison of methods for the typing of fish-pathogenic Vibrio spp. Systematic and Applied Microbiology. 1997;20(1):89-101.

5. Medini D, Donati C, Tettelin H, Masignani V, Rappuoli R. The microbial pan-genome. Curr Opin Genet Dev. 2005;15(6):589-94.

6. Hazen TH, Pan L, Gu JD, Sobecky PA. The contribution of mobile genetic elements to the evolution and ecology of Vibrios. Fems Microbiology Ecology. 2010;74(3):485-99.

7. Mclnerney JO, McNally A, O'Connell MJ. Why prokaryotes have pangenomes. Nat Microbiol. 2017;2(4).

8. Brockhurst MA, Harrison E, Hall JPJ, Richards T, McNally A, MacLean C. The Ecology and Evolution of Pangenomes. Curr Biol. 2019;29(20):R1094-R103.

9. Cohan FM. Transmission in the Origins of Bacterial Diversity, From Ecotypes to Phyla. Microbiol Spectr. 2017;5(5).

10. Jones JL. Chapter 11 - Vibrio. In: Dodd CER, Aldsworth T, Stein RA, Cliver DO, Riemann HP, editors. Foddborne Dieseases. 3 ed: Academic Press; 2017. p. 243-52.

11. Thompson CC, Vicente ACP, Souza RC, Vasconcelos ATR, Vesth T, Alves N, et al. Genomic taxonomy of vibrios. Bmc Evolutionary Biology. 2009;9.

12. Thompson FL, lida T, Swings J. Biodiversity of Vibrios. Microbiol Mol Biol R. 2004;68(3):403-31.

13. Hunt DE, David LA, Gevers D, Preheim SP, Alm EJ, Polz MF. Resource partitioning and sympatric differentiation among closely related bacterioplankton. Science. 2008;320(5879):1081-5.

14. Banks DJ, Beres SB, Musser JM. The fundamental contribution of phages to GAS evolution, genome diversification and strain emergence. Trends Microbiol. 2002;10(11):515-21.

15. Ohnishi M, Kurokawa K, Hayashi T. Diversification of Escherichia coli genomes: are bacteriophages the major contributors? Trends Microbiol. 2001;9(10):481-5.

16. Castillo D, Kauffman K, Hussain F, Kalatzis P, Rorbo N, Polz MF, et al. Widespread distribution of prophage-encoded virulence factors in marine Vibrio communities. Scientific reports. 2018;8.

17. Di Lorenzo M, Stork M, Tolmasky ME, Actis LA, Farrell D, Welch TJ, et al. Complete sequence of virulence plasmid pJM1 from the marine fish pathogen Vibrio anguillarum strain 775. J Bacteriol. 2003;185(19):5822-30.

18. Dunn AK, Martin MO, Stabb EV. Characterization of pES213, a small mobilizable plasmid from Vibrio fischeri. Plasmid. 2005;54(2):114-34.

19. Chibani CM, Hertel R, Hoppert M, Liesegang H, Wendling CC. Closely related Vibrio alginolyticus strains encode an identical repertoire of prophages and filamentous phages. bioRxiv 2019;859181.

20. Lukjancenko O, Ussery DW. Vibrio chromosome-specific families. Frontiers in Microbiology. 2014;5. 
21. Lee KK, Yu SR, Yang TI, Liu PC, Chen FR. Isolation and characterization of Vibrio alginolyticus isolated from diseased kuruma prawn, Penaeus japonicus. Lett Appl Microbiol. 1996;22(2):111-4.

22. Gonzalez-Escalona N, Blackstone GM, DePaola A. Characterization of a Vibrio alginolyticus strain, isolated from Alaskan oysters, carrying a hemolysin gene similar to the thermostable direct hemolysin-related hemolysin gene (trh) of Vibrio parahaemolyticus. Appl Environ Microbiol. 2006;72(12):7925-9.

23. Wendling CC, Piecyk A, Refardt D, Chibani C, Hertel R, Liesegang H, et al. Tripartite species interaction: eukaryotic hosts suffer more from phage susceptible than from phage resistant bacteria. BMC Evol Biol. 2017;17(98).

24. Wendling CC, Wegner KM. Relative contribution of reproductive investment, thermal stress and Vibrio infection to summer mortality phenomena in Pacific oysters. Aquaculture. 2013;412-413:88-96.

25. Poirier $M$, Listmann $L$, Roth 0 . Selection by higher-order effects of salinity and bacteria on early lifestages of Western Baltic spring-spawning herring. Evol Appl. 2017;10(6):603-15.

26. Le Roux F, Wegner K, Austin C, Vezzulli L, Osorio C, Amaro C, et al. The Emergence of Vibrio pathogens in Europe: Ecology, Evolution and Pathogenesis (Paris, 11-12 March 2015) Front Microbiol. 2015;6(830).

27. Wendling CC, Fabritzek AG, Wegner KM. Population-specific genotype x genotype x environment interactions in bacterial disease of early life stages of Pacific oyster larvae. Evol Appl. 2017;1(10).

28. Roth O, Keller I, Landis SH, Salzburger W, Reusch TB. Hosts are ahead in a marine host-parasite coevolutionary arms race: innate immune system adaptation in pipefish Syngnathus typhle against Vibrio phylotypes. Evolution. 2012;66(8):2528-39.

29. Pritchard L, Glover RH, Humphris S, Elphinstone JG, Toth IK. Genomics and taxonomy in diagnostics for food security: soft-rotting enterobacterial plant pathogens. Anal Methods-Uk. 2016;8(1):12-24.

30. Page AJ, Cummins CA, Hunt M, Wong VK, Reuter S, Holden MT, et al. Roary: rapid large-scale prokaryote pan genome analysis. Bioinformatics. 2015;31(22):3691-3.

31. Snipen L, Liland KH. micropan: an R-package for microbial pan-genomics. Bmc Bioinformatics. 2015;16:79.

32. Huerta-Cepas J, Szklarczyk D, Forslund K, Cook H, Heller D, Walter MC, et al. eggNOG 4.5: a hierarchical orthology framework with improved functional annotations for eukaryotic, prokaryotic and viral sequences. Nucleic Acids Res. 2016;44(D1):D286-D93.

33. Huerta-Cepas J, Szklarczyk D, Heller D, Hernandez-Plaza A, Forslund SK, Cook H, et al. eggNOG 5.0: a hierarchical, functionally and phylogenetically annotated orthology resource based on 5090 organisms and 2502 viruses. Nucleic Acids Res. 2019;47(D1):D309-D14.

34. Galperin MY, Makarova KS, Wolf YI, Koonin EV. Expanded microbial genome coverage and improved protein family annotation in the COG database. Nucleic Acids Res. 2015;43(D1):D261-D9.

35. Ronquist F, Teslenko M, van der Mark P, Ayres DL, Darling A, Hohna S, et al. MrBayes 3.2: Efficient Bayesian Phylogenetic Inference and Model Choice Across a Large Model Space. Syst Biol. 2012;61(3):539-42. 
36. Huelsenbeck JP, Ronquist F. MRBAYES: Bayesian inference of phylogenetic trees. Bioinformatics. 2001;17(8):754-5.

37. Tamura K, Nei M. Estimation of the Number of Nucleotide Substitutions in the Control Region of Mitochondrial-DNA in Humans and Chimpanzees. Mol Biol Evol. 1993;10(3):512-26.

38. Posada D, Buckley TR. Model selection and model averaging in phylogenetics: advantages of akaike information criterion and bayesian approaches over likelihood ratio tests. Syst Biol. 2004;53(5):793808.

39. Dhillon BK, Chiu TA, Laird MR, Langille MG, Brinkman FS. IslandViewer update: Improved genomic island discovery and visualization. Nucleic Acids Res. 2013;41(Web Server issue):W129-32.

40. Méhats $C$, Tanguy G, Paris B, Robert B, Pernin N, Ferré F, et al. Pregnancy induces a modulation of the cAMP phosphodiesterase 4-conformers ratio in human myometrium: consequences for the uterorelaxant effect of PDE4-selective inhibitors. Journal of Pharmacology and Experimental Therapeutics. 2000;292(2):817-23.

41. Alikhan NF, Petty NK, Ben Zakour NL, Beatson SA. BLAST Ring Image Generator (BRIG): simple prokaryote genome comparisons. Bmc Genomics. 2011;12:402.

42. Sullivan MJ, Petty NK, Beatson SA. Easyfig: a genome comparison visualizer. Bioinformatics. 2011;27(7):1009-10.

43. Chen L, Yang J, Yu J, Yao Z, Sun L, Shen Y, et al. VFDB: a reference database for bacterial virulence factors. Nucleic Acids Res. 2005;33(Database issue):D325-8.

44. Wickham H. ggplot2. Wires Comput Stat. 2011;3(2):180-5.

45. Okada K, lida T, Kita-Tsukamoto K, Honda T. Vibrios commonly possess two chromosomes. J Bacteriol. 2005;187(2):752-7.

46. Wang P, Wen Z, Li B, Zeng Z, Wang X. Complete genome sequence of Vibrio alginolyticus ATCC 33787( $T$ ) isolated from seawater with three native megaplasmids. Mar Genomics. 2016;28:45-7.

47. Goris J, Konstantinidis KT, Klappenbach JA, Coenye T, Vandamme P, Tiedje JM. DNA-DNA hybridization values and their relationship to whole-genome sequence similarities. Int J Syst Evol Microbiol. 2007;57(Pt 1):81-91.

48. Yoon SH, Ha SM, Lim J, Kwon S, Chun J. A large-scale evaluation of algorithms to calculate average nucleotide identity. Antonie Van Leeuwenhoek. 2017;110(10):1281-6.

49. Land M, Hauser L, Jun SR, Nookaew I, Leuze MR, Ahn TH, et al. Insights from 20 years of bacterial genome sequencing. Funct Integr Genomics. 2015;15(2):141-61.

50. Groisman EA, Ochman H. Pathogenicity islands: Bacterial evolution in quantum leaps. Cell. 1996;87(5):791-4.

51. Schmidt $H$, Hensel M. Pathogenicity islands in bacterial pathogenesis. Clin Microbiol Rev. 2004;17(1):14-+.

52. Vesth T, Wassenaar TM, Hallin PF, Snipen L, Lagesen K, Ussery DW. On the Origins of a Vibrio Species. Microbial Ecology. 2010;59(1):1-13. 
53. Shapiro BJ. How clonal are bacteria over time? Curr Opin Microbiol. 2016;31:116-23.

54. Kim HY, Yu SM, Jeong SC, Yoon SS, Oh YT. Effects of flaC Mutation on Stringent Response-Mediated Bacterial Growth, Toxin Production, and Motility in Vibrio cholerae. J Microbiol Biotechnol. 2018;28(5):816-20.

55. Chibani C, Hertel R, Goehlich H, Mertens V, Bunk B, Overmann J, et al. Complete Genome Sequence of Vibrio typhli K08M4, a fish pathogen isolated from the Kiel Fjord. in prep.

\section{Supplementary Information}

Table S1 Vibrio genomes accessed from NCBI 3.12.2019 used for PyANI analysis

Table S2 Genomic regions (GRs) unique for the nine $V$. alginolyticus strains from the Kiel-Fjord. For chromosome 1 (left) we used K10K4 as a reference, as this strain has more integrated prophages than the other Kiel isolates. For the same reason, we used strain K04M3 as a reference for chromosome 2. For a detailed comparison of GRs among all nine strains, see Figure 3 main manuscript.

Table S3 Functional characterization of all protein-coding sequences found on the Genomic Regions (using the eggNOG-mapper v2 software) with the resulting COG assignments. Similar to Table S2 we used strain K10K4 as reference for chromosome 1 and strain K04M3 as reference for chromosome 2. The column Kiel Specific indicates whether the GR is unique for the Kiel alginolyticus ecotype.

Table S4 Functional annotation of Kiel specific gene clusters (core genome [C], accessory genes [A], singletons [S], i.e. gene cluster which was only found within the Kiel alginolyticus ecotype with the resulting $C O G$ assignments.

Table S5 Resistance genes found using IslandViewer for all nine $V$. alginolyticus strains from the KielFjord. Even though this table shows the locus tags for strain K04M3 only, all other strains have been found to contain the exact same resistance genes.

\section{Table}

Due to technical limitations, table 1 is only available as a download in the supplemental files section.

Table 1 All $V$. alginolyticus strains used in the present study (* circular phage replicon).

\section{Figures}




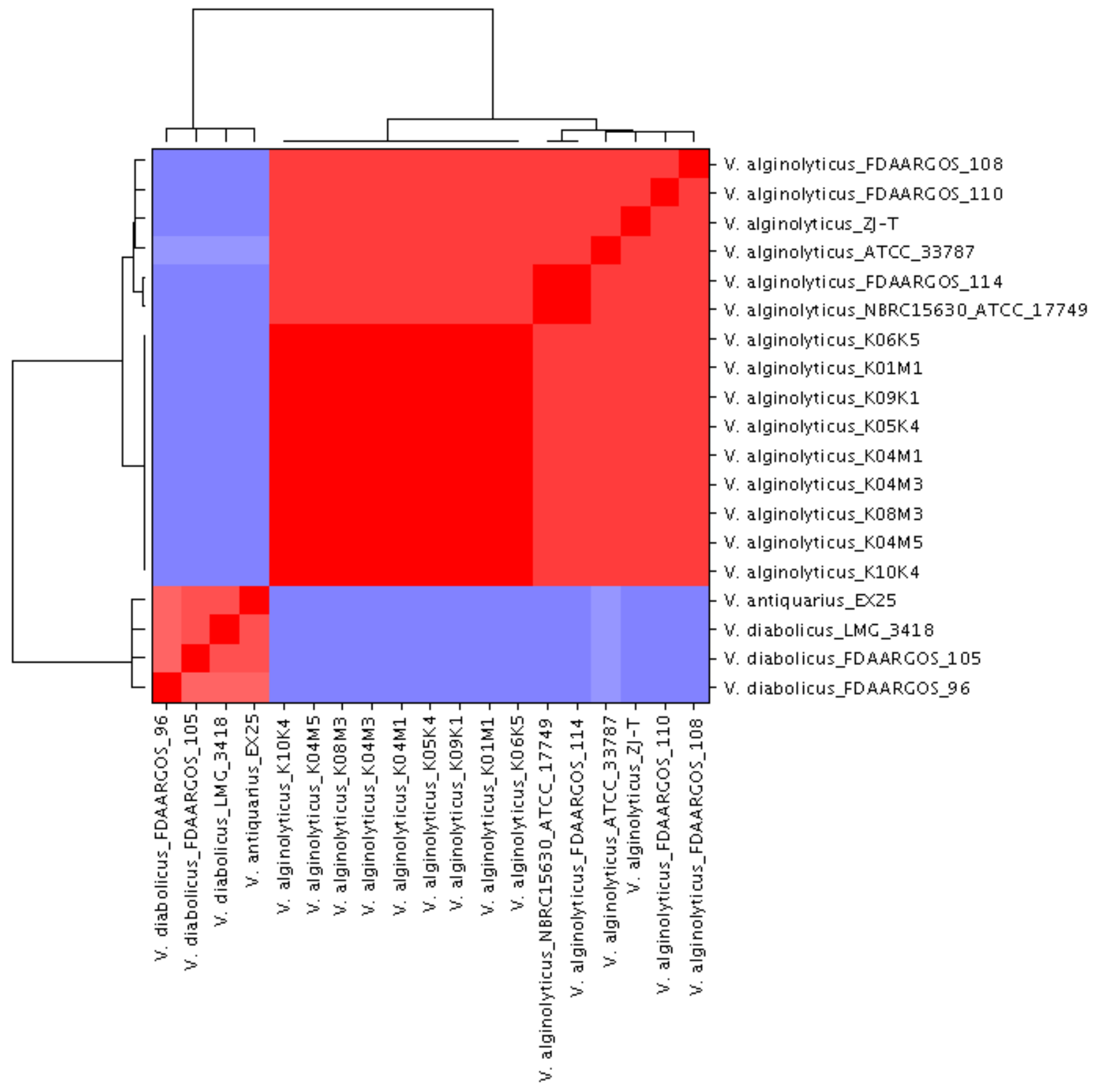

\section{Figure 1}

Average nucleotide identity (ANI) percentage analysis of closed Vibrio genomes. ANI analysis based on MUMmer alignment of the genome sequences was performed and visualized using PYANI. ANI-values ranging from 0 to $70 \%$ no hits, $70-95 \%$ ANI colored in blue and higher than $95 \% \mathrm{ANI}$ in red) All true V. alginolyticus (including the nine strains from the present study) can be separated from V. diabolicus and V. antiquaries (small red square, bottom left). 
(a)
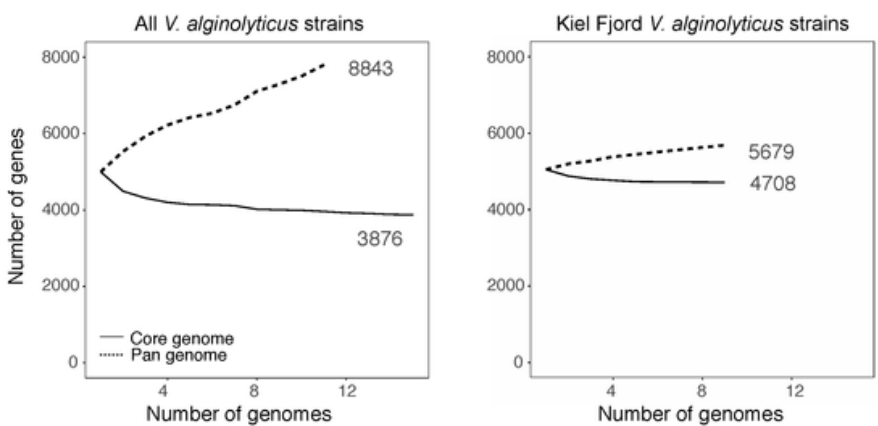

(b)
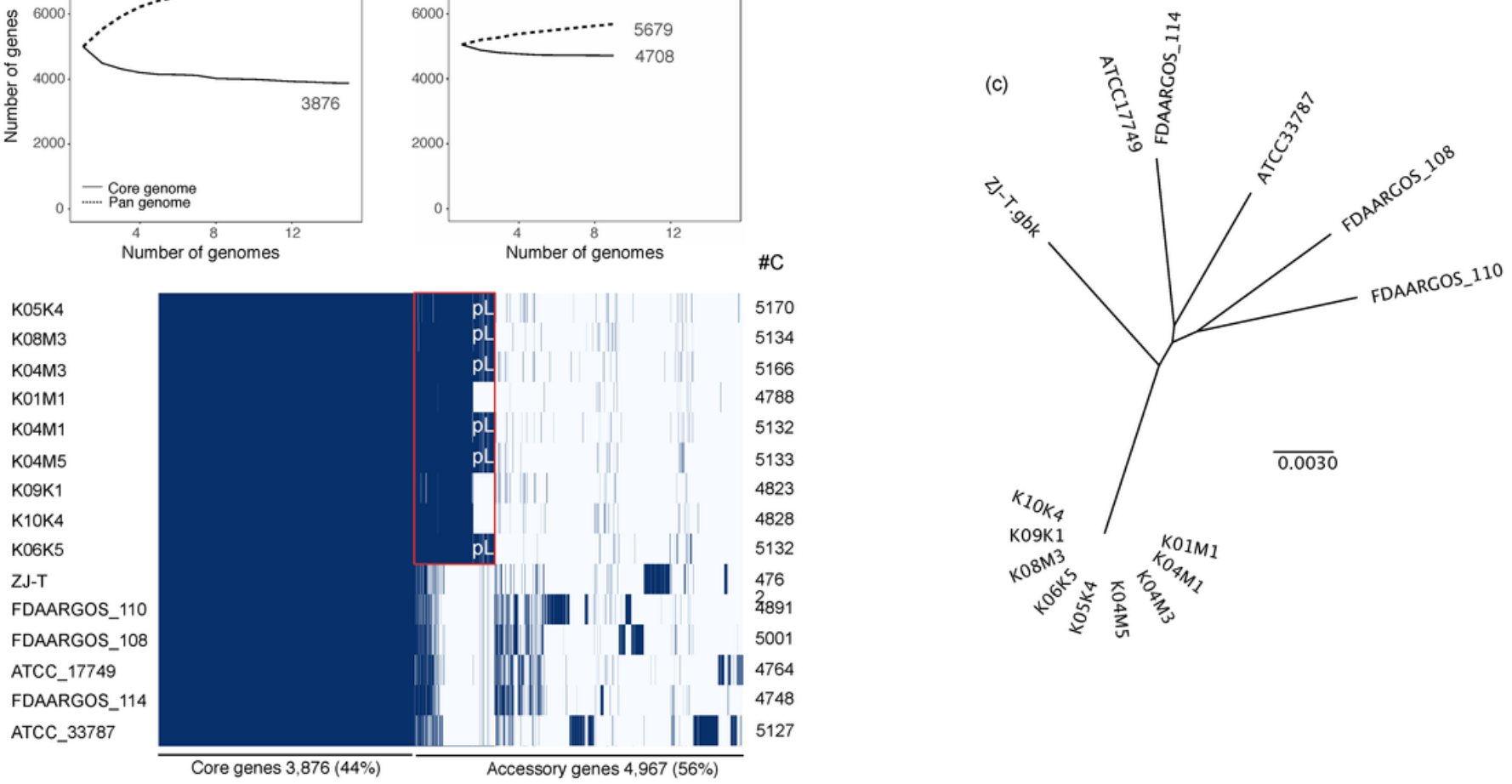

Figure 2

Pan-core-genome analysis of 15 closed Vibrio alginolyticus strains. (a) Number of gene clusters for pan (dashed line) and core genome (solid line) resulting from the sequential addition of genomes. The curve shows mean values of multiple iterations $(>100)$ where genomes were randomly added and the pan- and core-genome was calculated successively for all 15 steps (left: All $15 \mathrm{~V}$. alginolyticus strains) and nine steps (right: Kiel-Fjord V. alginolyticus only). (b) Gene presence (dark blue) -absence (light blue) matrix showing the distribution of core and accessory genes for each of the $15 \mathrm{~V}$. alginolyticus genomes. Each row corresponds to one strain, each column represents an orthologous gene family. Numbers on the right $(\# C)$ correspond to number of gene clusters per genome. The red square indicates the core-region specific for the Kiel isolates, the six mega-plasmids are indicated by white $\mathrm{pL}$ letters. (c) Bayesian phylogenetic tree of all 15 isolates based on the core-genome alignment. 

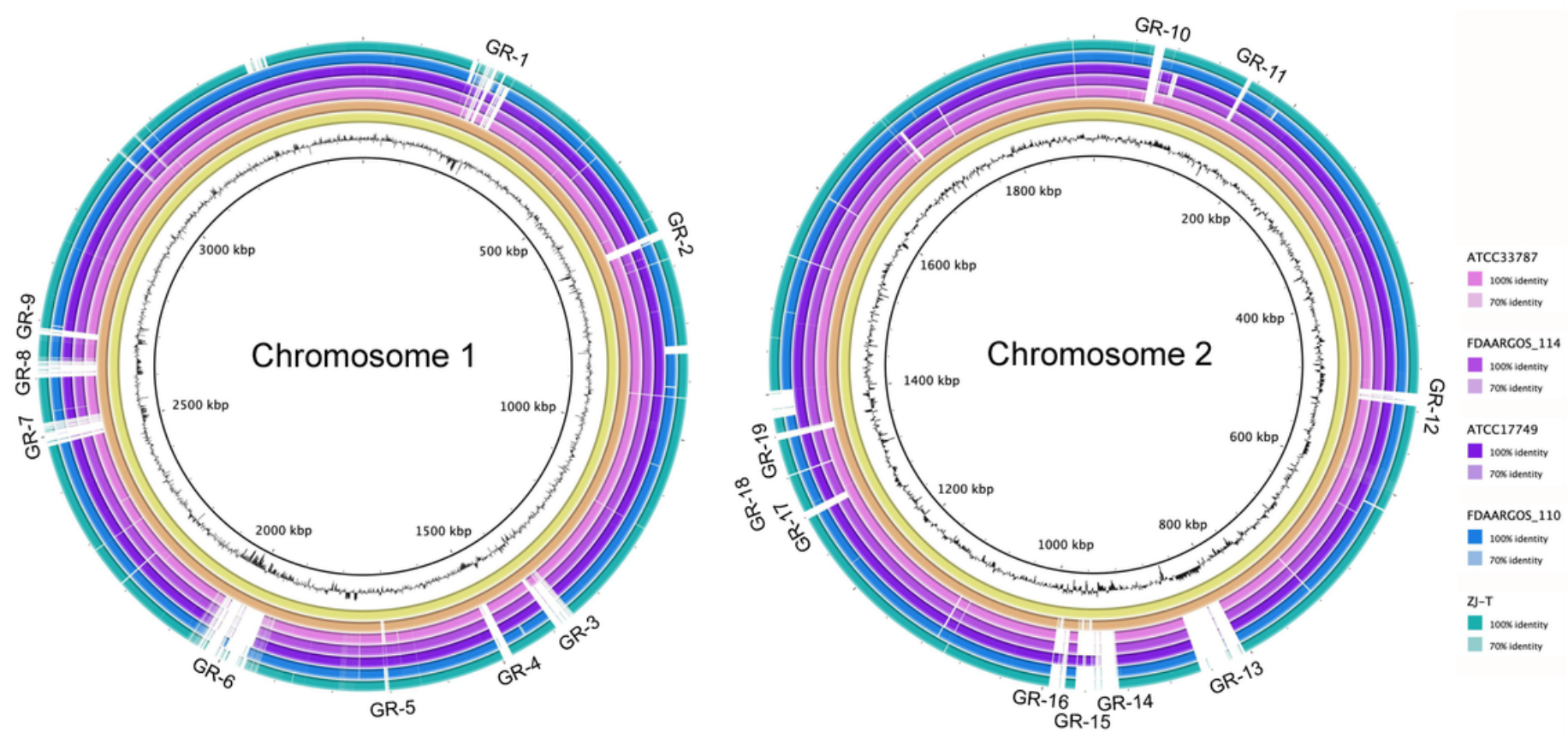

\section{Figure 3}

Comparison of all nine Kiel isolates and six other fully closed V. alginolyticus isolates. Blast matches between all strains are shown as concentric colored rings on a sliding scale according to percentage identity (100\% and 70\%). For chromosome 1 (left) we used K10K4 as a reference (inner black circle), as this strain has more integrated prophages than the other Kiel isolates. The second ring (yellow) corresponds to strain $\mathrm{K} 05 \mathrm{~K} 4$ which has the same integrated prophages as $\mathrm{K} 10 \mathrm{~K} 4$, the third ring (orange) corresponds to all other strains from Kiel, which miss GR 5, i.e. Vibrio phage VALGФ8. For the same reasons, we used strain K04M3 as a reference for chromosome 2. Here, the second ring (yellow) corresponds to strain K04M5, which has a similar prophage composition than K04M3 while the third ring (orange) corresponds to all other Kiel strains, which miss the multiphage-cassette consisting of repeats of both filamentous Vibrio phages VALGФ6 and Vibrio phage VALGФ8. GC content is shown in black. Genomic Regions (GRs) unique for Kiel isolates, which have additionally been predicted using IslandViewer are annotated on the outside.

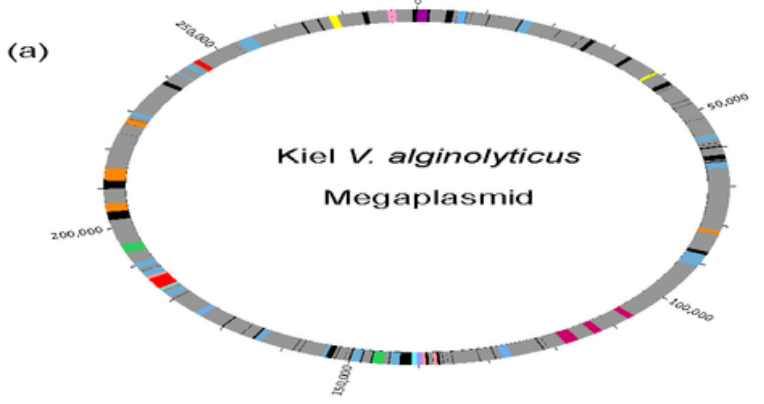

(b)

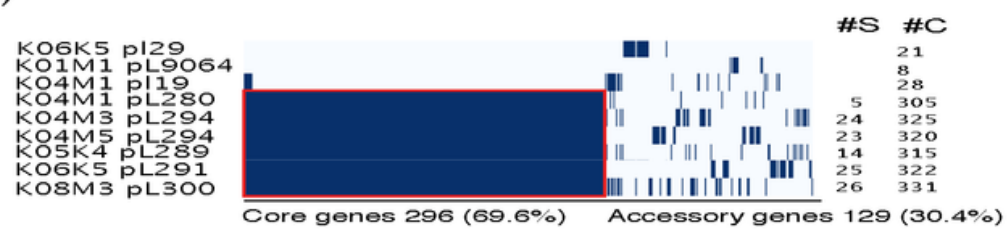

Figure 4 
Genomic variation among all nine Kiel V. alginolyticus strains. Left: Kiel-specific core genes (shared by all nine isolates), middle: Kiel-specific accessory genes (shared by two or more isolates, but not by all), and right: Kiel-specific singletons (unique for one isolate) distributed across replicons (CR1: chromosome 1, CR2: chromosome 2, Phages: extrachromosomal phages, Plasmids) and assigned to Clusters of Orthologous Groups of Proteins (COGs): black: information storage and processing; orange: cellular processes and signaling; blue: metabolism; green: poorly characterized; white: not assignable.

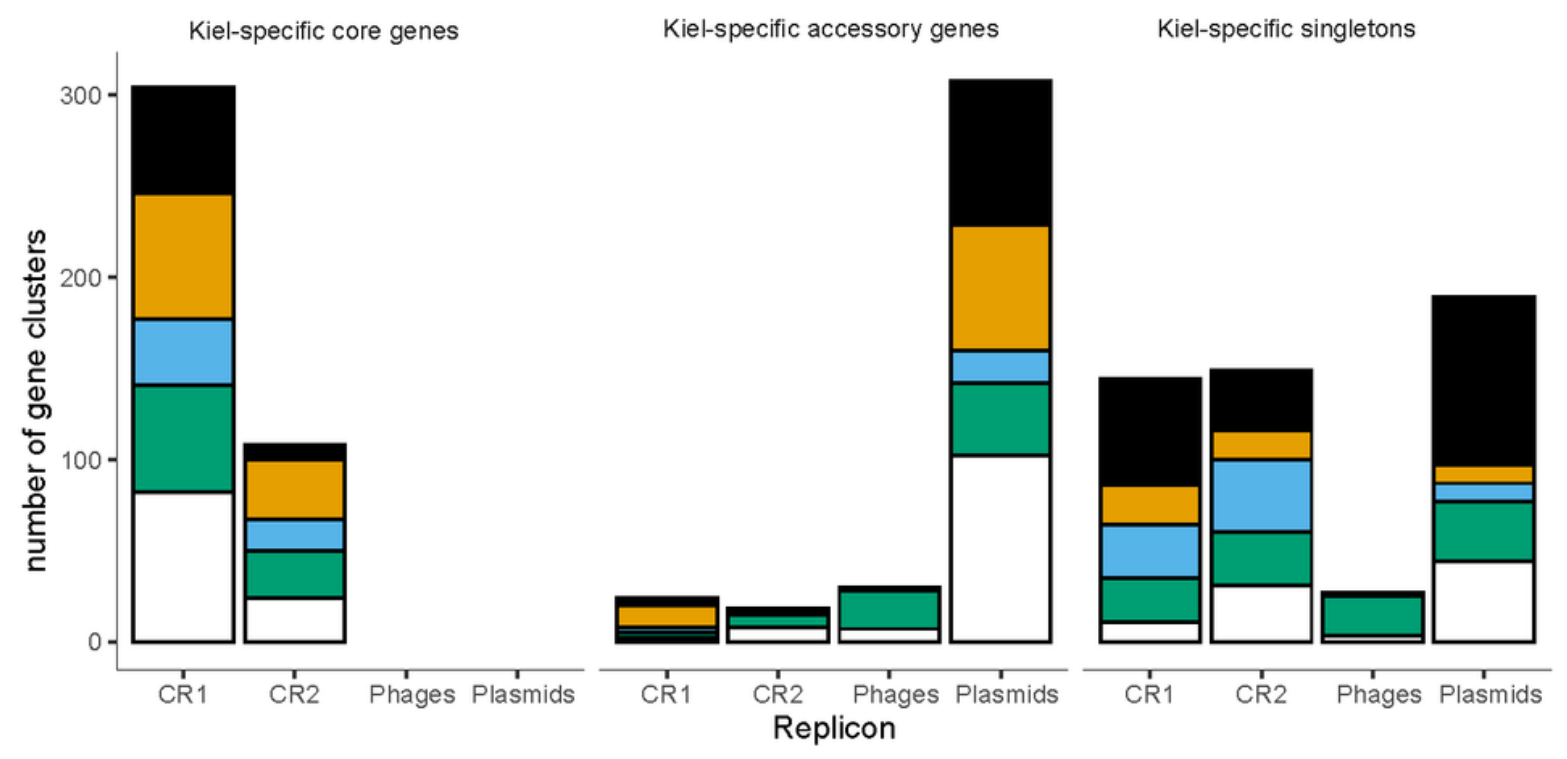

\section{Figure 5}

Comparative analysis of the Kiel alginolyticus mega plasmid. (a) Plasmid map. ORFs are color-coded according to COG functional categories: Purple: S, yellow: D, pink: EH, pink: K, blue: L, black: NA, magenta: $\mathrm{NU}$, red: O, light-blue: S, green: T, orange: U, sky-blue: V, grey: hypothetical proteins. (b) Gene presence (dark blue) -absence (light blue) matrix showing the distribution of core and accessory genes for each of the six mega-plasmids. Each row corresponds to one strain, each column represents an orthologous gene family. The red square represents the core genome of the mega-plasmids ( 296 genes) as opposed to the number of accessory genes of the mega-plasmids (129 genes). Numbers on the right correspond to (\#S) number of singletons per plasmid and (\#C) number of gene clusters per plasmid. 
(a)
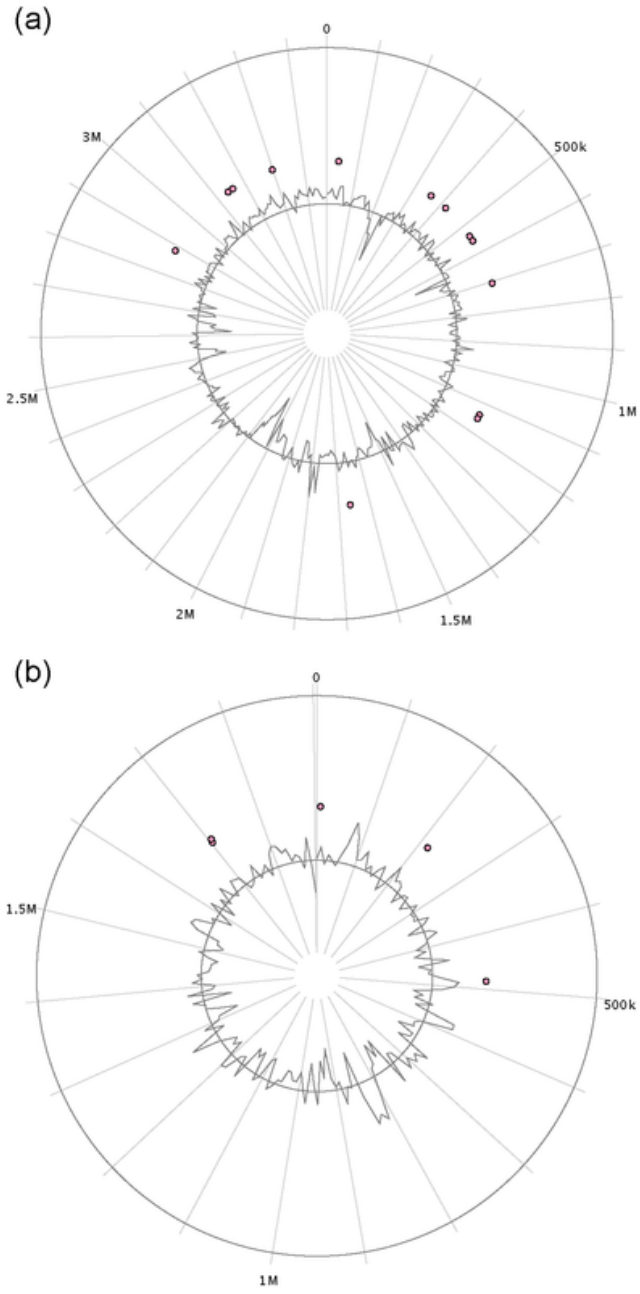

(c)

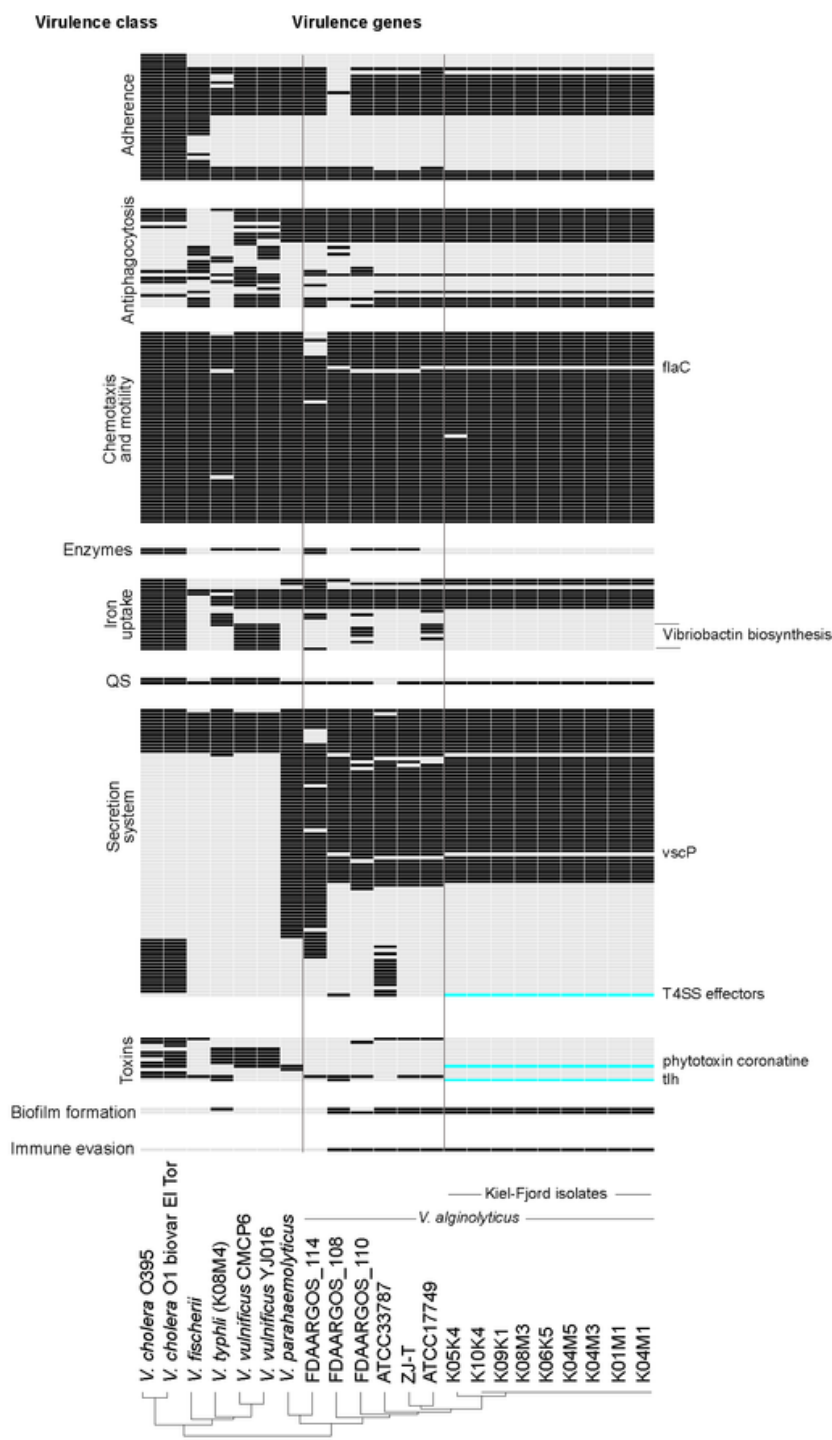

Figure 6

Resistance and virulence genes. Resistance genes are shown for (a) chromosome 1 and (b) chromosome 2. (c) Virulence genes, presented as a presence (black) / absence (grey) matrix, are grouped into 10 different virulence classes (left). Those virulence genes, which contribute to the unique profile of the Kielalginolyticus ecotype are labeled on the right and if present, marked in turquoise. Hierarchical cluster analysis of the 22 strains (bottom), which were incorporated into this analysis shows that the Kielalginolyticus ecotype can be separated from all other V. alginolyticus strains solely based on presence/ absence of specific virulence genes.

\section{Supplementary Files}


This is a list of supplementary files associated with this preprint. Click to download.

- TableS3.txt

- Tables1.txt

- Table1.txt

- Tables4.txt

- Tables5.txt

- Tables2.txt 